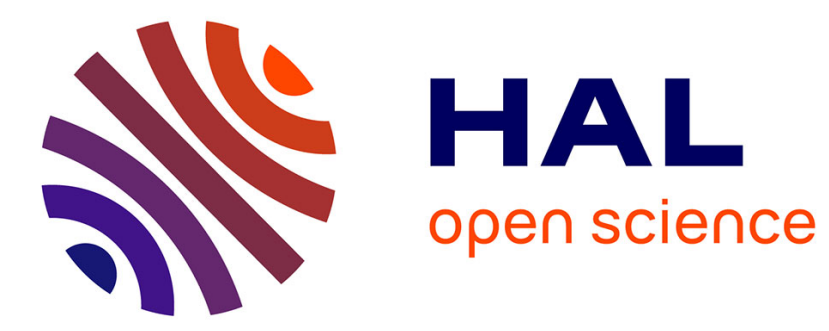

\title{
Design of a stable CW HCN laser for far infrared frequency synthesis
}

\author{
J.-M. Lourtioz, R. Adde
}

\section{To cite this version:}

J.-M. Lourtioz, R. Adde. Design of a stable CW HCN laser for far infrared frequency synthesis. Revue de Physique Appliquée, 1976, 11 (4), pp.533-540. 10.1051/rphysap:01976001104053300 . jpa00244088

\section{HAL Id: jpa-00244088 https://hal.science/jpa-00244088}

Submitted on 1 Jan 1976

HAL is a multi-disciplinary open access archive for the deposit and dissemination of scientific research documents, whether they are published or not. The documents may come from teaching and research institutions in France or abroad, or from public or private research centers.
L'archive ouverte pluridisciplinaire HAL, est destinée au dépôt et à la diffusion de documents scientifiques de niveau recherche, publiés ou non, émanant des établissements d'enseignement et de recherche français ou étrangers, des laboratoires publics ou privés. 


\title{
DESIGN OF A STABLE CW HCN LASER FOR FAR INFRARED FREQUENCY SYNTHESIS
}

\author{
J.-M. LOURTIOZ and R. ADDE \\ Institut d'Electronique Fondamentale*, Bâtiment 220, Université Paris XI, 91405 Orsay, France
}

(Reçu le 2 février 1976, accepté le 4 mars 1976)

\begin{abstract}
Résumé. - Nous décrivons la réalisation d'un laser HCN à la fois puissant et stable en fréquence, qui constitue ainsi un bon générateur pour la synthèse de fréquences infrarouges. L'influence des divers paramètres descriptifs du laser sur sa stabilité de fréquence et sa puissance de sortie a été analysée. Nous avons optimisé chacun des deux critères précédents en donnant la priorité à la stabilité de fréquence. Sur la raie d'émission à $891 \mathrm{GHz}(\lambda=337 \mu \mathrm{m})$, il est possible d'obtenir une stabilité de fréquence $(\Delta f \mid f)=5 \times 10^{-9}$ avec une puissance de sortie de $20 \mathrm{~mW}-30 \mathrm{~mW}$ lorsque le laser fonctionne en oscillateur libre. Sa puissance maximale est de $100 \mathrm{~mW}$.
\end{abstract}

\begin{abstract}
An HCN laser for infrared frequency synthesis has been designed to satisfy both conditions of high frequency stability and ouptut power. The influence of all the descriptive parameters of the laser on its frequency stability and power are analyzed. The optimization of the two above criteria is discussed with emphasis on frequency stability. On the $891 \mathrm{GHz}$ line the laser shows a natural frequency stability $(\Delta f \mid f)=5 \times 10^{-9}$ with an output power of $20 \mathrm{~mW}-30 \mathrm{~mW}$, and its maximum power is $100 \mathrm{~mW}$.
\end{abstract}

1. Introduction. - Submillimeter radiation sources with high frequency stability find one of their applications as transfer oscillators in the metrology of laser frequencies up to the visible wavelength region [1]. The development of such sources together with very high order harmonic mixers such as the Josephson junction has allowed substantial progress in far infrared frequency synthesis [2]. A high frequency stability and a sufficiently high output power are two requirements which are difficult to match simultaneously in the case of the $\mathrm{HCN}$ (or $\mathrm{H}_{2} \mathrm{O}$ ) gas lasers.

In this paper, we describe an $\mathrm{HCN}$ laser designed to satisfy both of the above conditions. This results from an optimization of the cavity and discharge laser parameters with passive techniques. On the $891 \mathrm{GHz}$ line, it shows a frequency stability $\Delta f / f \simeq 5 \times 10^{-9}$ with an output power of $20 \mathrm{~mW}-30 \mathrm{~mW}$ and its maximum output power is about $100 \mathrm{~mW}$. Most of the publications on the HCN laser are concerned with the enhancement of the output power and very few give indications on frequency stability and spectral purity. In this paper we focus more on these latter points. We analyze the influence of all the descriptive parameters of the laser on both its frequency stability and its power, and we discuss how to optimize the two conditions with emphasis on frequency stability.

The configuration selected for the laser cavity is conventional. It was preferred to the wave guide confi-

$\left(^{*}\right)$ Laboratoire associé au C. N. R. S. guration [3] which has not yet been tested from the spectral purity point of view. In these conditions, the achievement of a good cavity may be considered as an application in the far infrared of more general results on lasers. On the other hand, obtaining a quiet and efficient discharge implies a good understanding of the mechanisms specific to the $\mathrm{HCN}$ laser.

2. Fundamental mechanisms of the HCN laser. The identification of the emission lines of the HCN laser now seems well established. In 1967 Lide and Maki [4] proposed a mechanism based on the HCN molecules as emitting species. The strongest emission occurs at a wavelength $\lambda=337 \mu \mathrm{m}$ and they identified this emission with the rotational-vibrational transition

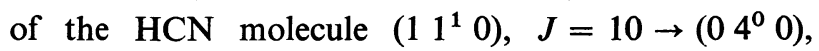
$J=9$ between states located at about $2800 \mathrm{~cm}^{-1}$ above the ground state. This explanation is supported by the experimental work of Hocker and Javan [5]. However, although similar studies completely determined the laser action process in the case of the water vapour laser [6], the precise mechanism of the excitation of the HCN stimulated emission is still unknown.

This results from the great complexity of the reactions which occur in the $\mathrm{HCN}$ laser. The emission lines are usually obtained in low pressure electrical discharges sustained in gas mixtures containing compounds of hydrogen, carbon and nitrogen such as $\mathrm{CH}_{4}-\mathrm{N}_{2}, \mathrm{CH}_{4}-\mathrm{NH}_{3}, \mathrm{CH}_{3} \mathrm{CN},\left(\mathrm{C}_{2} \mathrm{H}_{5}\right)_{2} \mathrm{O}+\mathrm{N}_{2}$, etc.

Pure cyanhydric acid is generally disregarded 
because of its excessive dissociation. Therefore the fundamental processes leading to the $\mathrm{HCN}$ stimulated emission are of two types :

- Mechanisms which give rise to the formation of the $\mathrm{HCN}$ molecules in the low pressure plasma :

a) Electronic collisions dissociating the initial gas mixture introduced in the discharge.

b) Chemical reactions between the free radicals created in the discharge. The following chemical reactions have been shown to take place when the discharge is sustained in $\mathrm{CH}_{4}-\mathrm{NH}_{3}$ or $\mathrm{CH}_{4}-\mathrm{NH}_{3}$ gas mixtures [7] :

$$
\begin{array}{ll}
\mathrm{CH}+\mathrm{N} \rightarrow \mathrm{HCN}, & \Delta H=-224 \mathrm{kcal} \\
\mathrm{CH}_{3}+\mathrm{N} \rightarrow \mathrm{HCN}+\mathrm{H}+\mathrm{H}_{2}, & \Delta H=-10 \mathrm{kcal} \\
\mathrm{CN}^{+}+\mathrm{H}_{2} \rightarrow \mathrm{HCN}+\mathrm{H}^{+} . &
\end{array}
$$

- Mechanisms leading to the excitation and population inversion of the $\mathrm{HCN}$ molecules.

a) The importance of the molecule-molecule type collisions seems to have been clearly demonstrated by Schötzau and Kneubühl [8]. Hydrogen (or deuterium) are the most important collision partners of the $\mathrm{HCN}$ molecules for laser emission :

$$
\mathrm{HCN}+\mathrm{H}_{2}^{*} \rightarrow \mathrm{HCN}^{*}+\mathrm{H}_{2} .
$$

They have shown that the laser intensity maximum corresponds to the optimum in the molecular hydrogen concentration and not to the optimum in the concentration of $\mathrm{HCN}$ molecules.

b) Lesieur and Kuntsreich have also demonstrated the chemical nature of the HCN laser [9]. Chemical reactions such as (1), (2), (3) may lead to the direct formation of HCN molecules in excited states.

c) Excitation mechanisms by electronic collision cannot be disregarded completely :

$$
\mathrm{HCN}+\mathrm{e}^{-} \rightarrow \mathrm{HCN}^{*}+\mathrm{e}^{-} \text {. }
$$

They remain valid for low energy electrons which do not lead to dissociative processes such as :

$$
\begin{aligned}
\mathrm{HCN}^{+}+\mathrm{e}^{-} & \rightarrow \mathrm{CN}^{+}+\underset{\downarrow}{\mathrm{H}}+2 \mathrm{e}^{-} \\
& \mathrm{H}_{2} \text { (molecular hydrogen recombination) }
\end{aligned}
$$

or

$$
\begin{aligned}
& \mathrm{HCN}+\mathrm{e}^{-} \rightarrow \mathrm{HCN}^{+}+2 \mathrm{e}^{-} \\
& \mathrm{HCN}+\mathrm{HCN}^{+} \rightarrow \mathrm{CN}+\mathrm{HCNH}^{+} .
\end{aligned}
$$

The efficiency of the above mentioned mechanisms strongly depends on the different descriptive parameters characterizing the discharge : gas mixture, total and partial gas pressures, discharge current $I$ and wall temperature of the discharge tube $T_{\mathrm{D}}$.

The discharge current, which is related to the electronic density of the plasma, determines the number of electron-molecule collisions and consequently the number of active chemical reactions. Therefore the laser intensity must be an increasing function of the discharge current. The wall temperature $T_{\mathrm{D}}$ and in second order the discharge current $I$ determine the neutral gas temperature of the plasma where the chemical reactions leading to the formation of the $\mathrm{HCN}$ molecules and their collision partners take place. Therefore an optimum temperature corresponding to the best efficiency of the chemical reactions exists. The pressure $\boldsymbol{P}$ determines the mean electron energy in the discharge as it gives the normalized electric field $E / P$, and there is an optimum pressure corresponding to the best distribution of the electronic energies.

3. Discussion of the cyanide laser parameters. The diagram in figure 1 shows how the descriptive parameters of the HCN laser may influence its frequency stability and output power level. These descriptive parameters are of two kinds, related either to the cavity or to the discharge and may influence the laser frequency stability and intensity via more fundamental quantities.

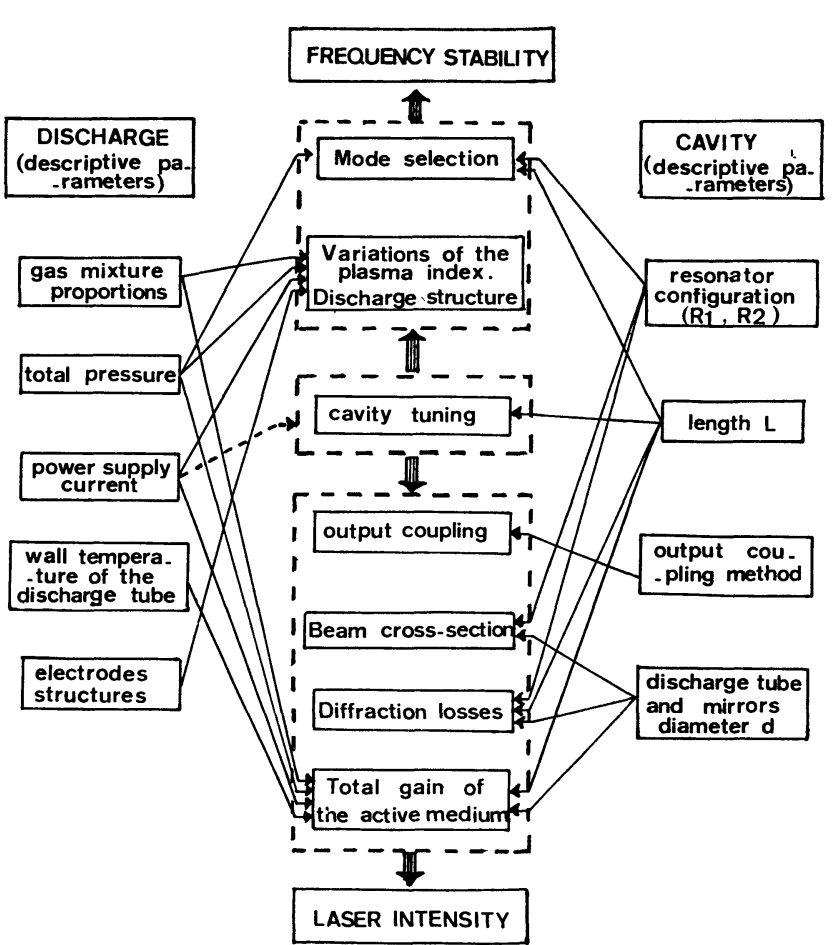

FIG. 1. - Influence of the descriptive parameters on the frequency stability and the intensity of the $\mathrm{HCN}$ laser.

3.1 Parameters Related to the fReQuency STABILITY AND THE SPECTRAL PURITY. - 3.1.1. Mode selection. - An essential condition for a laser source to be a good frequency generator is to oscillate only on one emission line and to run over only one of the resonator modes. The HCN laser and generally the submillimeter-wave lasers differ in this respect from the visible and I. R. lasers. The transitions relevant for the 
latter usually exhibit linewidths spread over several fundamental and many higher order modes and line separation may even be difficult. On the other hand, the cavity mode separation for submillimeter lasers such as the HCN laser is greater than the natural width of the laser line.

More precisely, single mode operation is realized if the minimum splitting $\Delta v_{M}$ between the main $\operatorname{TEM}_{q, m, n}$ cavity modes $(2 m+n \leqslant 3)$ is larger than the apparent emission linewidth $\Delta v_{\mathrm{app}}=\Delta v_{\mathrm{c}}+\Delta v_{0}$ where $\Delta v_{\mathrm{c}}$ is the free cavity bandwidth $(\simeq 0.3 \mathrm{MHz}$ in our case) and $\Delta v_{0}$ is the transition linewidth. Belland and Whitbourn [10] have shown that $\Delta v_{0}$ varies from $2.9 \mathrm{MHz}$ to $5.4 \mathrm{MHz}$ for the $\mathrm{HCN}$ laser when the total pressure increases from 0.1 torr to 1.1 torr and that homogeneous collision broadening replaces Doppler broadening $(\simeq 3 \mathrm{MHz})$ at high pressure. The splitting beetween modes $\Delta v_{M}$ depends on the resonator configuration and the cavity length $L$ [11]. In our design half confocal configuration, $L=6.50 \mathrm{~m}$ ) $\Delta v_{\mathrm{M}} \simeq 5 \mathrm{MHz}$ and single mode operation is naturally obtained. Therefore, tuning the laser resonator on the different modes is simply obtained by adjusting the resonator length $L$ (one of the cavity mirrors can easily be translated with a micrometer screw). In the same way, the strongest lines emitted in the CW HCN laser, $\lambda=337 \mu(891 \mathrm{GHz})$ and $\lambda=311 \mu(965 \mathrm{GHz})$ can easily be separated. Single mode operation is generally obtained for the $\mathrm{HCN}$ laser, but this condition is best fulfilled with lower pressure and a more selective resonator configuration. This is the case of confocal or half-confocal resonators.

3.1.2 Variations of the plasma index. - The most important short-term frequency instabilities are related to the fluctuations of the plasma index $n$ whose value is determined by the electronic density $n_{\mathrm{e}}$ of the plasma. The frequency instability resulting from a variation of the electronic density $\Delta n_{\mathrm{e}}$ (and consequently from a corresponding variation of the discharge current such that $\left.\Delta I / I=\Delta n_{\mathrm{e}} / n_{\mathrm{e}}\right)$ is given by :

$$
\frac{\Delta f}{f_{0}}=-\frac{\Delta n}{n}=\frac{\Delta n_{\mathrm{e}} \cdot e^{2}}{2 \varepsilon_{0} \cdot f_{0}^{2} \cdot M}
$$

where

$$
\varepsilon_{0}=\frac{1}{36 \pi} 10^{-9} \mathrm{MKSA}, \quad f_{0}=891 \mathrm{GHz},
$$

$e$ and $M$ are respectively the electronic charge and the electron mass, $\Delta f$ is the corresponding fluctuation of the laser frequency. Eq. (5) shows that the smaller the laser frequency $f_{0}$, the larger are the fluctuations related to the variations of the plasma index. This is a great disadvantage of the HCN laser as compared to higher frequency lasers; a $10 \%$ variation of the discharge current leads to a frequency variation of $6 \times 10^{-7}$, which means an active cavity linewidth of about $100 \mathrm{kHz}$.

REVUE DE PHYSIQUE APPLIQUÉE. - T. 11, No 4, JUILLET 1976
In the $\mathrm{HCN}$ laser, the fluctuations in the discharge current may have two origins :

- The main one is related to the fluctuations inherent to the three possible structures of the electric discharge : a stationary striated discharge, an oscillating striated discharge or an homogeneous glow discharge which is only obtained at high pressure ( $p>2$ torr), far beyond the optimum for the laser intensity.

- The other one is due to the instabilities of the discharge power supply. However, these instabilities will be always much smaller. Even a state of the art current power supply cannot prevent the important short term random fluctuations of the glow discharge plasma. Consequently, a good current stability implies operating with a stationary striated discharge. We shall see later that the running conditions providing the best current stability do not give the strongest laser intensity (weak pressure $p \lesssim 0.4$ torr, see 4 , Section 5.1 ).

3.1.3 Mechanical structure of the cavity. - The length fluctuations of the cavity give rise to the last frequency instability of the laser. The cavity thermal expansion induces a slow drift of the laser frequency. Consequently, the length of our laser cavity is determined using silica tube spacers (expansion coefficient $\Delta L / L=10^{-6} \%$ ) temperature controlled to $\mp 0.1^{\circ} \mathrm{C}$ with an internal water. The mechanical vibrations of the cavity lead to short and mean term cavity detuning. They have been highly reduced by setting the cavity on a heavy concrete table $(1500 \mathrm{~kg})$ isolated from ground vibrations by shock absorbers which cut out low frequencies below $4 \mathrm{~Hz}$.

3.2 Parameters Related to the LASER INTENSity. -3.2.1 Total gain of the active medium. - The gain of most submillimeter wave gas lasers is quite small, being of the order of $0.01-0.1 \mathrm{~m}^{-1}$. The total gain of the active medium is defined by the product $G_{0} . L$, where $G_{0}$ is the unsaturated gain per unit length (gain at zero intensity), constant along the positive column [10]. $G_{0}$ depends on the discharge parameter values (total and partial pressures, current $I$, wall temperature of the discharge tube $T_{\mathrm{D}}$ ) which determine the yield of the fundamental mechanisms described in section 2 .

$G_{0}$ may be also indirectly considered as depending on the discharge tube radius, which has been demonstrated experimentally [12] (in fact it is the saturation intensity of the active medium which provides such a dependence). Since the requirements for high frequency stability correspond to a low value of the gain per unit length (a few percent), we have selected a rather long cavity $(L=6.50 \mathrm{~m})$ in order to allow a large enough total gain. This choice is still compatible with single mode operation (see 3.1.1) and with mechanical design for a good frequency stability (see 3.1.3).

3.2.2 Diffraction losses. - The diffraction losses depend on the cavity length, the discharge tube or 
mirror diameters (via the Fresnel number) and the resonator configuration. They may be directly calculated with the diagrams given by $\mathrm{Li}$ [13]. Far infrared lasers may exhibit relatively important diffraction losses ( $>1 \%$ ) which consequently must be minimized. As the cavity length is already given (3.2.1) the calculated diffraction losses in figure 2 show that the

\begin{tabular}{|c|c|c|c|c|}
\hline $\begin{array}{l}\text { Resonator } \\
\text { configura- } \\
\text {-tion } \\
\end{array}$ & $\begin{array}{c}\begin{array}{c}\text { half_con } \\
\text { focal } \\
R_{1}=2 L, R_{2}\end{array} \\
\end{array}$ & $\begin{array}{c}\text { half _ con- } \\
\text {-centric } \\
R_{1}=L, R_{2}=\infty\end{array}$ & $\begin{array}{l}\text { ptene_plane } \\
R_{1}=R_{2}=0\end{array}$ & $\begin{array}{l}\text { confocal } \\
R_{\mathbf{1}}=R_{\mathbf{2}}=L\end{array}$ \\
\hline $\begin{array}{l}\text { Average of } \\
\text { the beam } \\
\text { cross-section }\end{array}$ & $15 \mathrm{~cm}^{2}$ & $20 \mathrm{~cm}^{2}$ & $\sim 60 \mathrm{~cm}^{2}$ & $6 \mathrm{~cm}^{2}$ \\
\hline $\begin{array}{c}\text { Diffraction } \\
\text { losses }\end{array}$ & $1 \%$ & $14 \%$ & $\sim 15 \%$ & $<0.1 \%$ \\
\hline $\begin{array}{l}\text { Beam diame } \\
\text { ter at the } \\
\text { output mirror }\end{array}$ & $4 \mathrm{~cm}$ & $1.6 \mathrm{~cm}$ & $\sim 7 \mathrm{~cm}$ & $3.4 \mathrm{~cm}$ \\
\hline $\begin{array}{l}\text { Beam diame } \\
\text { ter at the sphe } \\
\text { rical mirror }\end{array}$ & $5.2 \mathrm{~cm}$ & $8.6 \mathrm{~cm}$ & $\sim 7 \mathrm{~cm}$ & $3.4 \mathrm{~cm}$ \\
\hline
\end{tabular}

Nota : Beam cross-section and diameter are defined for a relative intensity of $1 / \mathrm{e}$ as compared to the intensity on the resonator axis.

Fig. 2. - Beam cross-section and diffraction losses for different resonator configurations of the $\mathrm{HCN}$ laser $(L=6.50 \mathrm{~m}$, $d=100 \mathrm{~mm}$ ).

resonator configurations providing the smallest diffraction losses are confocal $\left(L=R_{1}=R_{2}\right)$ or halfconfocal $\left(R_{1}=2 L, R_{2}=\infty\right)$ when a half-symmetric type is selected. The diffraction losses decrease with increasing diameter $d$ of the mirrors. On the other side, the laser gain decreases as the reciprocal of the discharge tube diameter [12]. Therefore, the value of $d$ must be a compromise ; for a half confocal resonator with a length $L=6.50 \mathrm{~m}$, the optimum diameter is found to be $\simeq 100 \mathrm{~mm}$.

3.2.3 Output coupling. - From the expression giving the saturation of the laser medium at the line centre [14], it can be shown that the power extracted from the laser is related to the different descriptive parameters of the cavity by [15] :

$$
P_{\text {ext }}=\frac{\mathcal{R} . I_{\mathrm{s}}}{\left\langle\frac{1}{S}\right\rangle}\left[\left(\frac{G_{0} L}{\mathcal{R}+D}\right)^{\alpha}-1\right] .
$$

The exponent $\alpha$ is equal to unity for an homogeneously broadened line and $\frac{1}{2}$ for a doppler-broadened line [14]. $I_{\mathrm{s}}$ is the saturation intensity of the active medium. $D$ is equal to the diffraction losses (in percent), $\langle 1 / S\rangle$ is the average of the reciprocal of the beam cross section in the cavity and $\mathcal{R}$ is the output coupling coefficient (in percent). Eq. (6) indicates that the optimum value of the coupling coefficient is given by:

$$
\mathcal{R}_{\mathrm{opt}}=\sqrt{G_{0} \cdot L \cdot D}-D, \text { if } \alpha=1 .
$$

The output coupling system which we have selected is a Michelson interferometer configuration with a polyethylene beam splitter and identical plane mirrors at the beam splitter end of the cavity.

The configuration previously described [16] allows a variable output coupling :

$$
\Re=4 r_{\|} t_{\|} \sin ^{2} \frac{2 \pi}{\lambda} \cdot\left(L_{2}-L_{1}\right)
$$

where $r_{\|}$and $t_{\|}$are respectively the reflection and transmission coefficients of the beam splitter for a parallel polarized beam, $\left(L_{2}-L_{1}\right)$ is the optical path between the two mirrors.

The dielectric beam splitter, which is set at an angle of $45^{\circ}$ off the laser axis, is a taut polyethylene membrane with a thickness providing constructive interference of the beam reflected from each of its surfaces. This thickness $e$ is an odd multiple of :

$$
e=\frac{1}{4} \lambda \cdot\left(n^{2}-\frac{1}{2}\right)^{-1 / 2} \text {. }
$$

In our experimental conditions, the output coupling may be varied beetween $0 \%$ and $14 \%$. This coupling method provides several advantages for the $\mathrm{HCN}$ laser :

- The coupling is continuously adjustable from zero to twice that of a simple beam splitter and the optimum coupling (7) may be easily reached.

- Mode distortion occurring with a coupling hole is minimized.

- The output beam is linearly polarized.

- As a result of eq. (8), the emission lines are easily identified.

3.2.4 Average cross-section $\langle S\rangle$ of the laser beam. - This is the last parameter acting on the laser intensity. It is mainly related to the selected resonator configuration. The larger $\langle S\rangle$, the greater is the number of HCN molecules which may interact in the lasing process and the larger is the laser intensity. The average cross section is obtained from expressions reported by Boyd and Kogelnik which give the characteristics of a gaussian beam in a laser cavity [11]. The table in figure 2 shows that an increase of the beam cross section by a factor of 4 when passing from a half confocal to a plane-plane configuration increases the diffraction losses for a large cavity by a factor of 15 .

Therefore, according to this result and to 3.1.1 and 3.2.2 the half confocal configuration appears to be most suitable for a cavity with such dimensions.

4. Technical description. - The laser schematic represented figure 3 shows the different parameters selected in sections 3.1 and 3.2 ; a half confocal cavity with length $L=6.50 \mathrm{~m}$, a diameter of mirrors and discharge tube $d=100 \mathrm{~mm}$, an output coupling consisting of a polyethylene beam splitter and Michelson interferometer.

The optimum gas mixtures which we have found for generating power are : $\mathrm{CH}_{4}-\mathrm{N}_{2}: 1-1$ and $\mathrm{CH}_{4}-\mathrm{NH}_{3}$ : 1-2. A good thermalization of the plasma and a good 


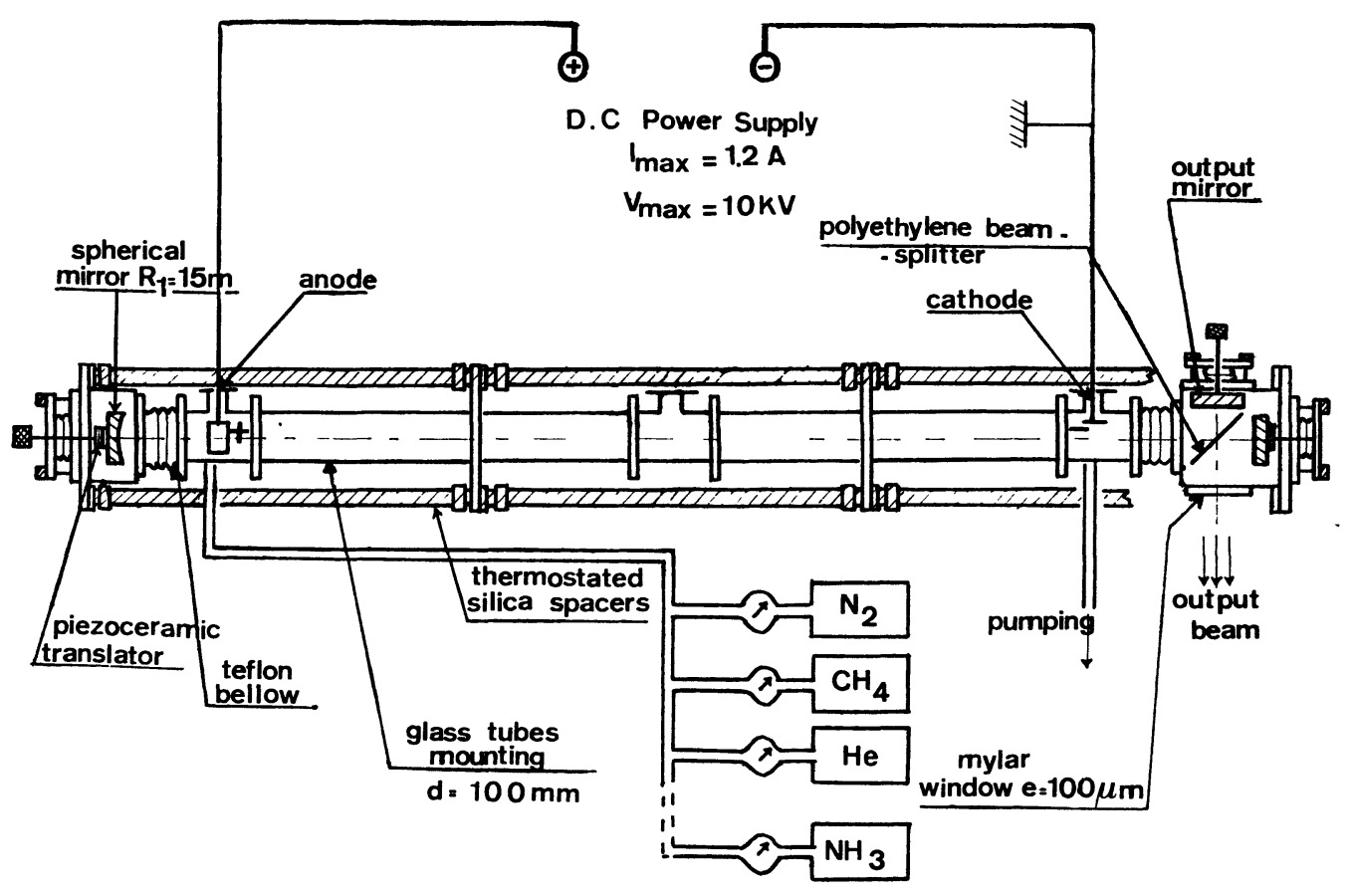

FIG. 3. - Schematic of the HCN laser $(L=6.50 \mathrm{~m})$.

homogeneity of the discharge may be obtained advantageously by the addition of helium to the above gas mixtures.

In our design, gases flow in at the anode level and are pumped out at the cathode level or through the cathode itself. The total flow rate was not found to have a critical value ; in our conditions it was typically about $200 \mathrm{~cm}^{3} \mathrm{~min}^{-1}$ at S. T. P.

The power supply allows a maximum d. c. voltage of $10 \mathrm{kV}$ with a maximum d. c. current $1.2 \mathrm{~A}$. Typical discharge conditions are $V=3800 \mathrm{~V}, I=0.8 \mathrm{~A}$. For such current and voltage values a suitable cathode is a watercooled stainless steel hollow cathode. The anode may have simple shapes (stainless steel ring coaxial with the discharge tube, brass tube transverse to the discharge axis), but an arcing point is commonly observed on the anode, even with pressure and current conditions providing a good discharge stability. To cancel these noisy oscillations, it is necessary to operate with a better design [1] which provides a well defined equipotential plane and gives rise to a more stationary striated discharge.

5. Experimental results. - 5.1 STABILITY OF THE DISCHARGE. - We have investigated the influence of the gas mixtures, partial and total pressures and current value on the discharge current stability. Figure 4 clearly shows the three discharge structures commonly observed and which correspond to the following three pressure-current domains :

- In the first region ( $p<0.4$ torr) the discharge is said to be very stable. Very stable striations extend from the cathode to the anode and just the arcing

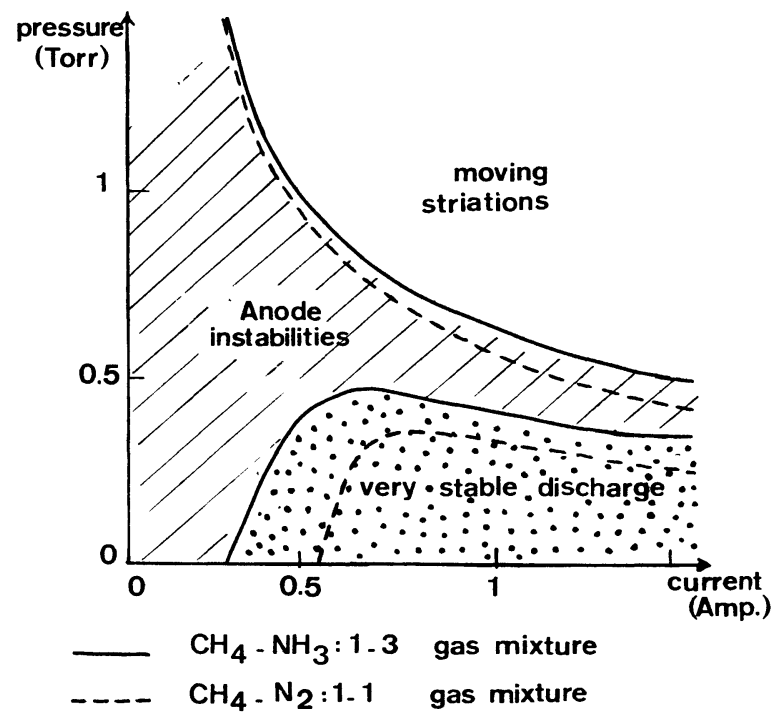

FIG. 4. - Influence of the pressure-current conditions on, the discharge structure.

point on the anode may still be found to oscillate. In the best situation the resulting stability is then limited by the current stability of the power supply $\left(\simeq 10^{-3}\right)$, and in the general case current fluctuations do not exceed a few $10^{-3}$. The laser power which may be extracted in such running conditions is about 20-30 $\mathrm{mW}$.

- In the second region, moving striations appear near the anode while stationary striations still exist in the main part of the glow discharge on the cathode side. The resulting stability of the discharge current is found to be about a few per cent and the number of moving striations increases with increasing pressure. 
- In the third region, moving striations occur in the whole discharge and current instabilities may reach $10 \%$ of the current value.

The best gas mixtures for reaching a good discharge stability are $\mathrm{CH}_{4}-\mathrm{N}_{2}: 1-1$ and $\mathrm{CH}_{4}-\mathrm{NH}_{3}: 1-3$, but the latter gas mixture shows slightly better performances. The addition of helium always improves the discharge stability ; the current fluctuations may be decreased by a factor of 2 and higher pressures of $\mathrm{CH}_{4}-\mathrm{N}_{2}$ or $\mathrm{CH}_{4}-\mathrm{NH}_{3}$ gas mixtures may be used without reaching the moving striation domain. However in our conditions too large an amount of helium in the discharge reduces the laser intensity. By cooling the neutral gas in the plasma, it decreases the efficiency of the chemical reactions described in section 2 .

It should be noted that all these results concerning the discharge stability may vary with other discharge geometries (electrodes design, discharge tube dimensions) because of the complexity of the discharge glow striations [17] and their dependence upon any discharge parameter. However, variations in the geometry are generally found to give second order effects.

5.2 LASER INTENSITY. - Here we report the results concerning the influence of the descriptive parameters of the discharge on the laser intensity (Care has been taken to vary only one parameter at a time and keep the others constant). A calibrated Moll thermopile is used for the measurements of the laser output power and gives a voltage of $1 \mathrm{mV}$ for a radiated power of $6 \mathrm{~mW}$. No important change has been observed in these measurements when running for about $200 \mathrm{~h}$. The tube pollution by polymer deposits does not strongly affect the Laser power and the different influences of the discharge parameters. The power output just slowly decreases with pollution of the polyethylene beam-splitter) $20 \%$ decrease after $200 \mathrm{~h}$ running).

5.2.1 Pressure dependence. - As we reported in section 2, for given discharge current and wall temperature of the discharge tube, there is an optimum value of the gas pressure. From the data in figure 5 it can be seen that this optimum increases with the current taken as a parameter. The optimum values are situated between 1 torr and 1.5 torr, whatever is the gas mixture. These results are in good agreement with other published data [18] [19]. The maximum output power which was obtained is about $100 \mathrm{~mW}$.

5.2.2 Current dependence. - The data in figure 6 shows that the laser intensity is an increasing function of the discharge current ; first, it strongly increases with the discharge current up to $I \simeq 1 \mathrm{~A}$, then increases more slowly for higher values. According to other published measurements [18] [19], it seems that the optimum current value is near $2 \mathrm{~A}$. Therefore, we may expect to further increase our laser output power which is at present limited by the power supply current
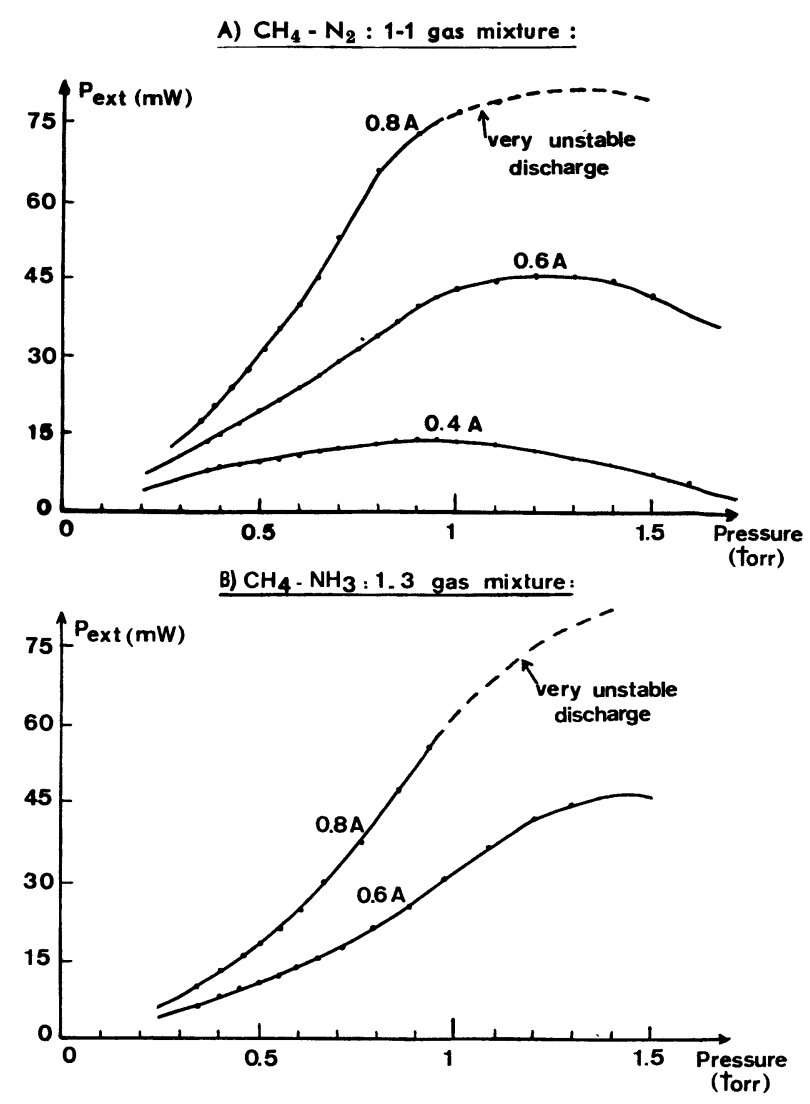

Fig. 5. - Pressure dependence of the HCN laser intensity.

A) $\mathrm{CH}_{4} \cdot \mathrm{N2} 2: 1.1$ gas mixture:

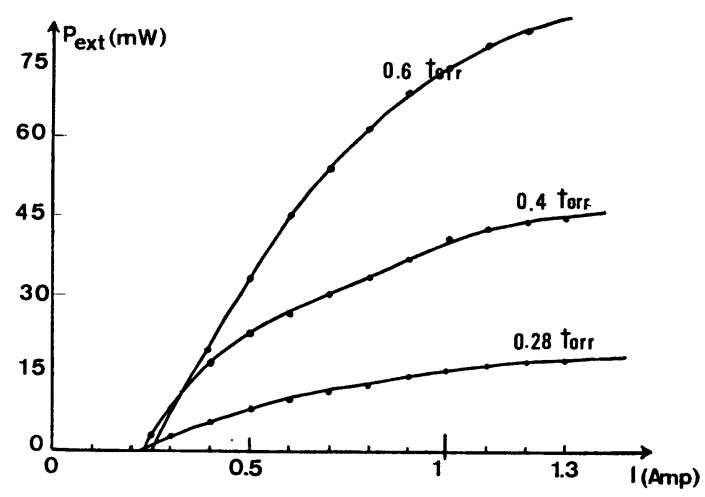

B) $\mathrm{CH}_{4} \cdot \mathrm{NH}_{3}: 1.3$ gas mixture:

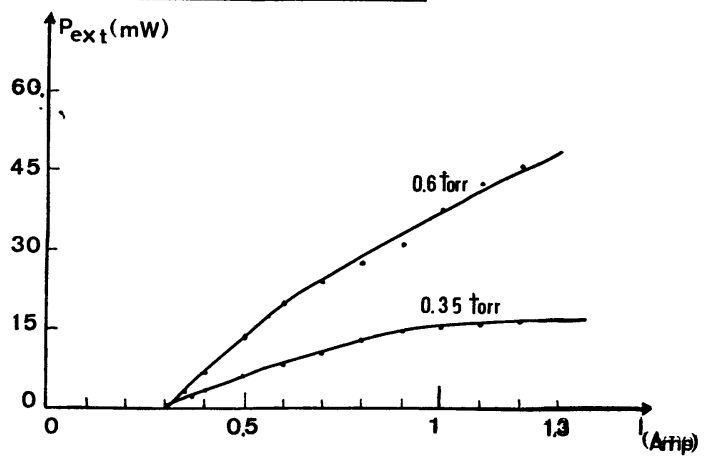

Fig. 6. - Discharge current dependence of the HCN laser intensity. 
(1.2 A) and by heating effects in the cathode region (operation with a high discharge current necessitates working with a hot cathode design [20]).

Note : The curves of figure 6 were obtained with gas mixtures giving the best discharge stability. For different partial pressures $\mathrm{CH}_{4}-\mathrm{NH}_{3}: 1-2$ the same performances were obtained with the two gas mixtures.

5.2.3 Dependence on the temperature of the discharge tube $T_{\mathbf{D}}$ - - In figure 7, the laser intensity is drawn as a

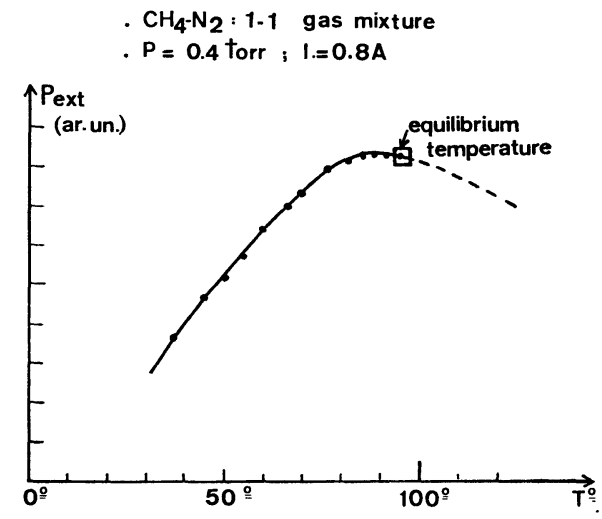

FIG. 7. - Discharge tube temperature dependence of the HCN laser intensity.

function of the wall temperature $T_{\mathrm{D}}$ of the discharge tube. This result emphasizes the importance of this parameter [20]. The laser intensity increases by a factor of 3 when the discharge tube temperature goes from $25^{\circ} \mathrm{C}$ (ambient temperature) to the equilibrium temperature resulting from thermal exchanges with the external surroundings. The temperature of the discharge tube settles naturally in the neighbourhood of the optimum temperature $\left(T_{\mathrm{D}} \simeq 100^{\circ} \mathrm{C}\right)$ corresponding to the selected gas mixtures. This result is in good agreement with the previous work of Belland and Veron [20].

5.3 LASER FREQUENCY STABILITY. - Measurements of the spectral purity of the laser beam have been performed using a Josephson harmonic mixer [15]. The $891 \mathrm{GHz}$ is mixed in a superconducting $\mathrm{Nb}-\mathrm{Nb}$ point contact with the 26th harmonic of a $34 \mathrm{GHz}$ klystron or the 96th harmonic of a $9.28 \mathrm{GHz}$ klystron. The klystrons are phased-locked on a MOS-5 stabilizer, and their frequency measured with a frequency counter. Details of operation concerning the Josephson harmonic-mixer will be published in a forthcoming paper. Thei.f. signal centreed at $150 \mathrm{M} \mathrm{Hz}$ from the harmonic mixer is amplified and displayed on a spectrum analyzer. The measured spectral width of the $\mathrm{HCN}$ laser operating at $891 \mathrm{GHz}$ as a free oscillator is $\leqslant 4 \mathrm{kHz}$ when the conditions of frequency stability of the laser are optimized. This corresponds to a frequency stability $\simeq 5 \times 10^{-9}$.

6. Conclusions. - The experimental results clearly demonstrate the influence of the different descriptive parameters of the laser analyzed in section 3 . The discharge conditions optimizing the $\mathrm{HCN}$ laser frequency stability are different from the conditions which optimize its output power. To illustrate this point, we have reported in figure 8 the power which may be extracted

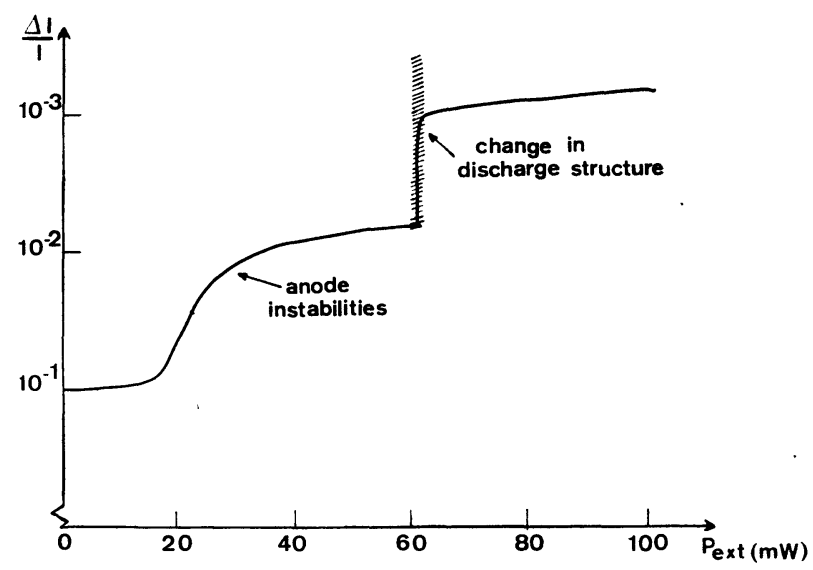

FIG. 8. - Output power of the HCN laser $(337 \mu \mathrm{m})$ as a function of the discharge current stability.

from the laser for a given level of current fluctuations. In our experimental set-up using only passive techniques of stabilization, a power level of 20-30 mW may be delivered at $891 \mathrm{GHz}$ when current fluctuations are reduced to nearly $10^{-3}$. A better current stability would require a much more sophisticated current power supply. The power levels and frequency stability which have been measured are in good agreement with the performances obtained at NBS [1] and NPL [21] for a free running $\mathrm{HCN}$ laser.

\section{References}

[1] WeLls, J. S., IEEE Transactions on instrumentations and measurements 22 (1973) 113.

[2] McDonald, D. G., Risley, A. S., Cupp, J. D., Evenson, K. M. and Ashley, J. R., Appl. Phys. Lett. 20 (1972) 296.

[3] Belland, P., Thèse d'Etat, Université de Paris VI (1975).
[4] Lide, D. R., Maki, J. R. and A. G., Appl. Phys. Lett. 11 (1967) 2.

[5] Hocker, L. O., Javan, A., Phys. Lett. 25A (1967) 489.

[6] Pichamutu, J. P., Hassler, J. C. and Coleman, P. D., Appl. Phys. Lett. 19 (1971) 410.

[7] Toupance, G., Thèse d'Etat, Université de Paris VI (1973). 
[8] Schötzau, H. J., MahleR, H. J. and KNeubühl, F. R., Phys. Lett. (1972) 286.

[9] Kunstreich, S. and Lesieur, J.P., Opt. Commun. 13 (1975) 17.

[10] Belland, P., Ciura, A. I., Whitbourn, L. B., Opt . Commun 11 (1974) 21.

[11] Boyd, G. D. and Kogelnik, H., Bell. Syst. Techn. J. (1962) 1348.

[12] Birch, J. R., Bradley, C. C., Infrared Phys. 13 (1973) 99.

[13] LI, T., Bell. Syst. Techn. J. (1965) 918.

[14] Chang. W. S., Principles of quantum Electronics (Addison Wisley New York).
[15] LourTioz, J.-M., Thèse de Docteur Ingénieur, Université de Paris XI (1975).

[16] Bradley, C. C., Infrared. Phys. 12 (1972) 287.

[17] Oleson, N. L., COOPER, A. W., Advances in electronics and electron physics (Ed. Marton) 24, 1968.

[18] Gehre, O., Techn. Note. IPP IV/39, Max Planck Institute (1972).

[19] VolK, R., Phys. Lett. 42A (1972) 321.

[20] Belland, P. and Veron, D., Opt. Commun. 9 (1973) 146.

[21] Blaney, T. G., Knight, D. J. E., Appl. Phys. 6(1973) 936. 